\title{
Integration of Projection Profile Matching into Clinical MR Scanner System for Real-Time Organ Tracking and Image Registration
}

\author{
Junichi Tokuda ${ }^{1}$, Masaya Hirano ${ }^{2}$, Tetsuji Tsukamoto ${ }^{2}$, Takeyoshi Dohi ${ }^{1}$, and \\ Nobuhiko Hata ${ }^{1}$ \\ 1 Graduate School of Information Science and Technology, The University of Tokyo, \\ 7-3-1, Hongo, Bunkyo-ku, Tokyo 113-8656, Japan, \\ \{junichi, dohi, noby\}@atre.t.u-tokyo.ac.jp, \\ http://www.atre.t.u-tokyo.ac.jp/ \\ ${ }^{2}$ GE Yokogawa Medical Systems Ltd., \\ 4-7-127, Asahigaoka, Hino-shi, Tokyo 191-8503, Japan, \\ \{masaya.hirano, tetsuji.tsukamoto\}@gemsa.med.ge.com
}

\begin{abstract}
We propose integrating projection profile matching into a 1.5 Tesla clinical magnetic resonance (MR) scanner system to track a target organ in real-time for MRI guided therapy. As soon as a scanner acquires echoes, MR echo data are transferred immediately from the scanner to an "on-the-fly" processing computer that executes projection profile matching, image reconstruction, image registration and motion correction, The system provides respiratory motion information about a target organ with a frame rate of up to $10 \mathrm{~Hz}$ and delay time of 200 ms. We report measurements of a phantom and a volunteer to evaluate the delay and the accuracy of the motion measurement with respect to the true motion, and feasibility. The study compares projection profile matching-based measurement and a "golden-standard" obtained by tracking a phantom with a video camera. The error was $1.5 \mathrm{~mm}$ with a delay of $200 \mathrm{~ms}$. We tracked the moving liver of a volunteer, and two studies demonstrates that the system can be applied to clinical use.
\end{abstract}

\section{Introduction}

Among various imaging modalities, magnetic resonance imaging (MRI) is a superior tool, not only for diagnosis but also for guidance in therapy, because of its physiological imaging capabilities that enable soft tissue discrimination and detailed delineation of anatomic features. Wide applications of MRI guided therapy have been proposed in recent years, such as MRI guided biopsy [1], cryotherapy [2], the monitoring of local drug administration 3]. Others examples include thermal ablation therapy, such as interstitial laser induction 4, as well as in radio-frequency (RF)- [5], microwave- [6] and ultrasound- 7] ablation.

Inatraoperative MRI can provide valuable information for locating, targeting and monitoring energy deposition; however, image quality, imaging time and delay are still critical constraints on interactive guidance. To complement the 
quality and scan time of intraoperative MRI, Gering proposed a visualization system using image fusion of pre-surgical high quality diagnostic images and intraoperative MR images 8. The important feature of this system was that optical tracking was used to provide location information for surgical tools. The location information was updated every $100 \mathrm{~ms}$, and enabled interactive guidance. However, managing organ motion caused by respiration was still difficult, so patients were required to hold their breath during therapy.

As a solution for this problem, we reported projection profile matching for fast image registration in intraoperative MR imaging [9]. Projection profile matching is a method of estimating the displacement of a target organ by using "navigator echoes" [10. In previous study, echo data were stored in a buffer memory until the end of the scan, and were processed retrospectively. At that time, we could only report that this process works well in the case of artifact reduction, but retrospective processing is meaningless for using navigator echoes as guidance information. To be used effectively, echo processing must be performed immediately after echo acquisition and provide target motion information in real-time. The objectives of the current study are to integrate projection profile matchingbased fast image registration in a 1.5 Tesla clinical scanner system and validate this method in a clinically realistic setting. We report the engineering setup for immediately transferring echo data online after acquiring an echo and performing matching "on-the-fly". This new addition enables us to validate the accuracy and speed of the image registration by using both a phantom and a volunteer, and to use both in real-time and online. Therefore, the engineering contribution of this study is a newly proposed system setup for migrating fast image registration in the MR scanner. This study further introduces the clinical significance of this new setup, in which the organ can be tracked during the intraoperative MRI scan thus enabling motion compensation in MR imaging for RF-, microwave, or laser-induced coagulation therapy.

\section{Materials and Methods}

\subsection{Projection Profile Matching}

A detailed account of projection pfofile matching can be found in 9 . In twodimenstional gradient echo sequence, the signal received by the radio-frequency $(\mathrm{RF})$ coil is:

$$
\begin{aligned}
\int_{x} \int_{y} m(x, y) \exp \left\{-j\left(\gamma G_{x} x t+\gamma G_{y} y T_{y}\right)\right\} d x d y \\
=\int_{x} \int_{y} M(x, y) \exp \left\{-j\left(k_{x} x+k_{y} y\right)\right\} d x d y=S\left(k_{x}, k_{y}\right)
\end{aligned}
$$

where $G_{x}$ and $G_{y}$ is the magnetic field gradient along frequency-encoded and phase-encoded directions, $\gamma$ is the gyromagnetic ratio, $t$ is the time from the start of echo acquisition, $T_{y}$ is the duration of $G_{y}, k_{x}=\gamma G_{x} t$, and $k_{y}=\gamma G_{y} T_{y}$. 


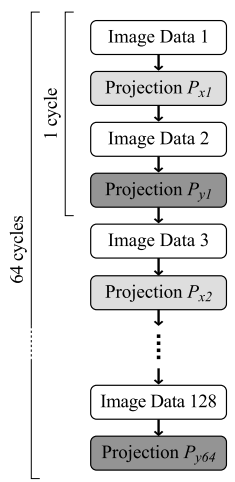

Fig. 1. A sequence to acquire both imaging echoes and navigator echoes. Total of 64 projection profiles per each axis were obtained during one image acquisition.

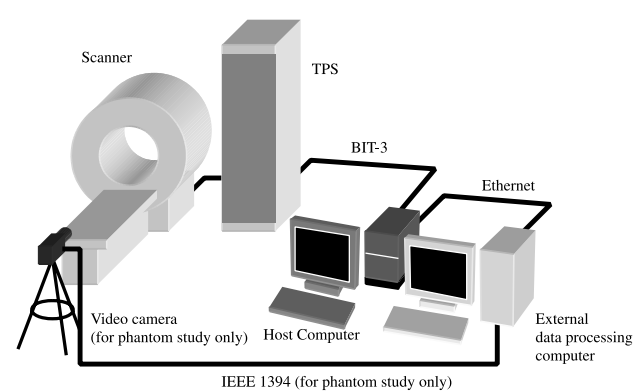

Fig. 2. Configuration of "on-the-fly" projection profile matching system. Echo data received in the scanner is immediately transferred to the external computer via Transceiver, Processing and Storage (TPS) computer and host computer. In phantom study, digital video camera was connected to the external computer to provide the "golden-standard" of phantom motion.

When $k_{y}=0$, a Fourier transformation of $S\left(k_{x}, k_{y}\right)$ becomes a projection profile of $m(x, y)$ onto the $\mathrm{x}$ axis:

$$
p_{x}(x)=\int_{-\infty}^{\infty} m(x, y) d y .
$$

Assuming that an object in the field of view is rigid, we can estimate the 2dimensional translation of the object from a 1-dimensional translation of the projection profile. Therefore an echo without phase encoding $\left(k_{y}=0\right)$ is called a "navigator echo". We matched the projection profiles before and after the motion by maximizing a cross-correlation of two profiles. Motion tracking using a projection profile, that can be produced from one echo, is obviously much faster than motion detection using an intraoperative image which requires hundreds of echoes.

\subsection{Pulse Sequence}

We developed a sequence to acquire simultaneously navigator and imaging echoes by basing on the gradient echo sequence. The customized sequence has a total of 256 pulse excitation and echo acquisition steps per image (Fig. 11). One hundred twenty-eight steps are phase-encoded signals used to reconstruct an image, while the other 128 steps are used to acquire signals to obtain the navigator echo described in the previous section. Scan parameters, such as repetition time (TR), echo time (TE), flip angle, field of view (FOV), etc. were configurable. The time resolution of projection profile matching based tracking depends on TR, and is formulated as $\frac{1}{T R \times 4} \mathrm{~Hz}$. Also, the imaging time depends on TR, and is $T R \times 256 \mathrm{~s}$. 


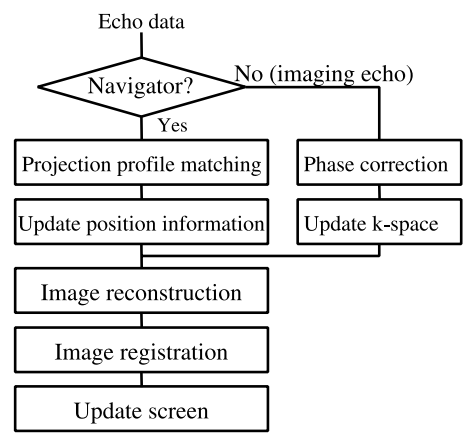

Fig. 3. Flow diagram shows process steps in the external data processing computer. A current target position is stored, then updated as each navigator echo arrives. This position information is used in phase correction for artifact reduction and image registration process.

\subsection{Real-Time Tracking and Image Registration System}

We developed a real-time tracking and image registration system running with a 1.5 Tesla clinical MR scanner (Signa MR/i, GE Medical Systems, Milwaukee, WI). The real-time "on-the-fly" echo processing program runs on an external data processing workstation (OS: RedHat Linux 7.3, CPU: Pentium IV 2.8GHz, Memory: 512MB PC1066 RIMM), which was connected to the host computer of the MR scanner system via a 100 Base-T Ethernet (Fig. 21). The normal reconstruction process that runs on a Transfer, Processing and Storage (TPS) computer of the MR scanner system was reconfigured, to transfer echo signal data to the external workstation immediately after echo acquisition.

The real-time "on-the-fly" echo-processing program (Fig. 3) receives two types of data: imaging echoes and navigator echoes. On arrival of echo data at the external computer, the program recognizes the data type by the header information, and performs projection profile matching when the echo is a navigator echo, or updates the k-space by applying phase correction to reduce motion artifacts if the echo is an imaging echo.

After these steps, 2-D fast Fourier transformation (FFT) is applied to the $\mathrm{k}$-space to reconstruct an image, and image registration is performed using displacement information calculated by projection profile matching. The registered image and the displacement of the target organ are displayed on a console screen connected to the external workstation. The screen is updated after each processing of echo data, therefore an image update rate of $\frac{1}{T R}$ frames per second is achieved.

\section{$3 \quad$ Experiments}

\subsection{Phantom Study}

First we conducted a phantom study to evaluate the proposed system in terms of accuracy and delay of motion detection and image registration. We moved a cylindrical phantom (160 mm diameter) manually into the scanner's bore, and tracked the displacement of the phantom by simultaneously using projection profile matching and a digital video camera, that was installed outside the bore. 
Errors between the projection profile matching based tracking and the video tracking as a "golden-standard" were calculate after the scans. We also evaluated the delay of the projection profile matching based tracking from by comparing these results to the camera images.

The cylindrical phantom was placed on the patient bed in the bore. The axis of the phantom was kept in parallel with the axis of the scanner's bore while the phantom was moving, and the phantom movement was constrained only in the plane perpendicular to the bore's (or phantom's) axis. The imaging slice was also perpendicular to the axis, therefore, the MR image of the phantom looked like a moving disk in the imaging slice.

The digital video camera (DCR-PC110, Sony Corporation, Japan) was placed on the axis of the scanner's bore (Fig. 2). The distance between the base of the phantom and the camera was 5 meters. Because the camera's field of view was about $54 \times 36 \mathrm{~cm}$ at the phantom's base, and the image resolution was $720 \times 480$ pixels, 1 pixel movement in a video image was equivalent to about $0.75 \mathrm{~mm}$. We performed template matching retrospectively on each video frame to measure the "golden-standard" displacement of the phantom at each frame. The results of projection profile matching based tracking were compared with this video tracking after the scan.

The video images were transferred to the external workstation via an IEEE1394 connection (transfer rate of $400 \mathrm{Mbps}$ ), with a frame rate of 29.97 frames per second, and were stored in the external workstation. The storing time was determined using the workstation's clock and recorded with the images. The delay of projection profile matching based tracking from the true phantom motion $\left(D_{P}\right)$ was the amount of the time shift between the trajectories measured by projection profile matching and video camera tracking $\left(T_{V P}\right)$ and the delay of video camera images $\left(D_{V}\right) . T_{V P}$ was determined after the scans by maximizing the cross correlation of the two trajectories.

The scan parameters were $\mathrm{TR}=100 \mathrm{~ms}, \mathrm{TE}=8.4 \mathrm{~ms}$, and FOV=350mm. Unfortunately the $\mathrm{TR}$ was constrained by the connection between the scanner and the external data processing computer.

\subsection{Volunteer Study}

Second, to evaluate the feasibility of the system we used it to study a volunteer's liver. To measure the displacement of the liver, we assumed that the liver moves mostly along the superior-to-inferior axis during respiration. This assumption is supported by Blackall's work [11] showing that the mode of variation consists primarily of a rigid-body translation in the superior-to-inferior direction, which accounts for a large percentage of the total variation. Before starting projection profile matching, we scanned the baseline liver image while the volunteer held his breath, segmented the image to isolate the liver region, and generated a baseline projection profile. We used only the liver region as the baseline image. Because we had no "golden-standard" for the liver's position, we only compared this result to the volunteer's respiratory phase, which was detected by the MR system's respiratory monitor. 


\section{Results}

\subsection{Phantom Study}

The $D_{V}$ was $100 \mathrm{~ms}$ (corresponding to 3 frames of video image), and the mean $T_{V P}$ was $100 \mathrm{~ms}$. Therefore, we determined the $D_{P}$ was $200 \mathrm{~ms}$. We also evaluated the error between projection profile matching and video tracking. Because the projection profile matching and video frames were not synchronized, we calculated the displacement at the corresponding time point by interpolating video tracking results, and evaluated the errors of displacement at each time point. The root mean square (RMS) error was $1.5 \mathrm{~mm}$ in the $\mathrm{x}$ direction and $2.0 \mathrm{~mm}$ in the $\mathrm{y}$ direction, and standard deviation of the error was $0.94 \mathrm{~mm}$ in $\mathrm{x}$ direction and $1.17 \mathrm{~mm}$ in the $\mathrm{y}$ direction.

\subsection{Volunteer Study}

Fig. (4) compares the time series of the position of the volunteer's liver as measured by projection profile matching and by recording his respiration. Also, screenshots are shown in Fig. 5. The translation of the liver and respiration had strong correlation, and the result indicates that projection profile matching provided reasonable information of liver translation in real-time.

\section{Discussion}

We have developed and evaluated the first prototype of real-time motion tracking and image registration embedded in a clinical MRI system. The phantom study showed that the method can track a moving phantom every $400 \mathrm{~ms}$ for each direction with an RMS error of $1.5 \mathrm{~mm}$ and a delay of $200 \mathrm{~ms}$. Note that the time resolution of position estimation depends on TR. Because we developed the prototypes by modifying a high level layer of a clinical system and that system inherently restricted the transfer rate of the echo data, TR shorter than $80 \mathrm{~ms}$ caused transfer errors. Tracking with time resolution of tens milliseconds would be possible, if the transfer rate is improved.

The short matching time and high time-resolution of displacement measurement of projection profile matching indicate that the method offers promise as non-invasive real-time organ motion tracking for automated targeting, especially in MRI-guided robotics [12], and focused ultrasound surgery (FUS) [13]. Clinical MRI can provide the information for locating, targeting and monitoring energy deposition, but Jolesz stated that whole body, organ, and tissue motion are still constraints on achieving precise treatment.

Our future plans are to extend the degree of freedom (DOF) for motion estimation. Welch presented a basic study of a more generalized navigator echo method called spherical navigator echo (SNAV), which samples the data in a spherical shell of k-space, for six DOF rigid body motion estimation [14. The implementing SNAV in intraoperative MRI is a challenging issue because the 

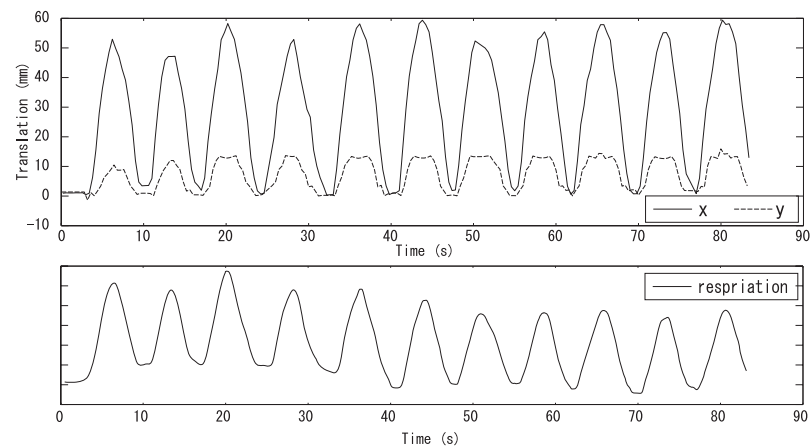

$t_{1}$
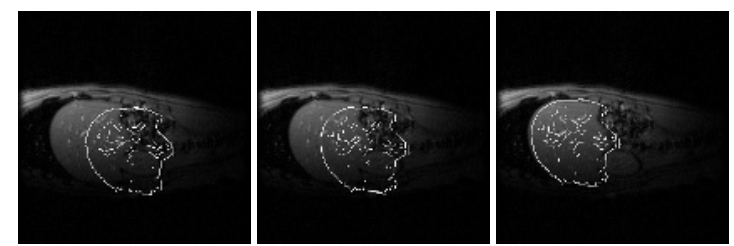

b
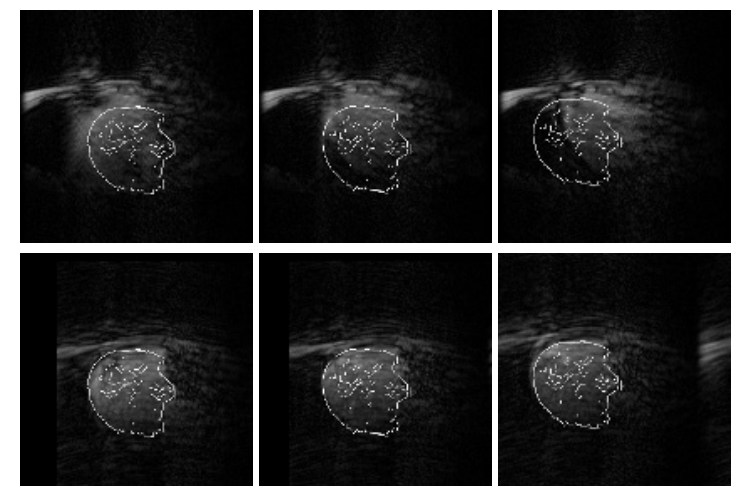

Fig. 4. Comparison between position of a volunteer's liver measured by projection profile matching (upper) and respiration phase (lower).

Fig. 5. Screenshots at three time points $t_{1}, t_{2}$ and $t_{3}$. Projection profile matching was performed in real-time on the volunteer's liver image during deep breathing. Edge image of liver in a baseline image was registered at real-time position and overlaid on same baseline image (a), real-time liver images without phase correction / registration (b), realtime liver image with phase correction followed by image registration(c). Position of liver in images in row (b) is indistinguishable, while liver image is clearly shown in images in row (c).

intraoperative MRI has low contrast, and low signal-to-noise ratio. Deformation detection is another important issue for using this system for surgical guidance.

In conclusion, we proposed embedding a real-time organ tracking and image registration technique in intraoperative MRI, and demonstrated its feasibility. The study led us to believe that the proposed method extends the application of intraoperative MRI guided surgery.

Acknowledgements. This study was funded by a Grant-in-Aid for Scientific Research (A-14702070 and B-13558103) from the Ministry of Education, Culture, Sports, Science and Technology, Japan. N. Hata's work for this study was supported by grants from the Suzuken Memorial Foundation, Kurata Grants, and the Toyota Physical and Chemical Research Institute. 


\section{References}

1. Silverman, S. G., Collick, B. D., Figueira, M. R., Khorasani, R., Adams, D. F., Newman, R. W., Topulos, G. P., Jolesz, F. A.: Interactive MR-guided biopsy in an open-configuration MR-imaging system. Radiology 197 (1995) 175-181

2. Tacke, J., Adam, G., Speetzen, R., Brucksch, K., Bucker, A., Heschel, I., Prescher, A., van Vaals, J. J., Hunter, D. W., Rau, G., Gunther, R. W.: MR-guided interstitial cryotherapy of the liver with a novel, nitrogen-cooled cryoprobe. Magnet. Reson. Med. 39 (1998) 354-360

3. Lu, D. S. K., Sinha, S., Lucas, J., Farahani, K., Lufkin, R., Lewin, K.: MR-guided percutaneous ethanol ablation of liver tissue in a .2-T open MR system: Preliminary study in porcine model. J. Magn. Reson. Im. 7 (1997) 303-308

4. Vogl, T. J., Mack, M. G., Roggan, A., Straub, R., Eichler, K. C., Muller, P. K., Knappe, V., Felix, R.: Internally cooled power laser for MR-guided interstitial laser-induced thermotherapy of liver lesions: Initial clinical results. Radiology 209 (1998) 381-385

5. Lewin, J. S., Connell, C. F., Duerk, J. L., Chung, Y. C., Clampitt, J. Spisak, G. S. Gazelle, M. E., Haaga, J. R.: Interactive MRI-guided radiofrequency interstitial thermal ablation of abdominal tumors: Clinical trial for evaluation of safety and feasibility. J. Magn. Reson. Im. 8 (1998) 40-47

6. Morikawa S., Inubushi, T., Kurumi, Y., et al.: MR-guided microwave thermocoagulation therapy of liver tumors: Initial clinical experiences using a $0.5 \mathrm{~T}$ open MR system. J. Magn. Reson. Im. 16 (2002) 576-583

7. Graham, S. J., Chen, L., Leitch, M., Peters, R. D., Bronskill, M. J., Foster, F. S., Henkelman, R. M., Plewes, D. B.: Quantifying tissue damage due to focused ultrasound heating observed by MRI. Magnet. Reson. Med. 41 (1999) 321-328

8. Gering, D. T., Nabavi, A., Kikinis, R., Hata, N., O'Donnell, J., Wells III, W. M.: An Integrated Visualization System for Surgical Planning and Guidance Using Image Fusion and an Open MR. J. of Magn. Reson. Im. 13 (2001) 967-975

9. Hata, N., Tokuda, J., Morikawa, S., Dohi, T.: Projection Profile Matching for Intraoperative MRI Registration Embedded in MR Imaging Sequence. Medical Image Computing and Computer-Assisted Intervention - MICCAI 2002, LNCS 2489 (2002) 164-169

10. Ehman, R. L., Felmlee, J. P.: Adaptive Technique for High Definition-MR Imaging of Moving Structures Navigator Echoes. Radiology 173 (1989) 255-263

11. Blackall, J. M., King, A. P., Penney, G. P., Adam, A., Hawkes, D. J.: A statistical model of respiratory motion and deformation of the liver. Medical Image Computing and Computer-Assisted Intervention - MICCAI 2001, LNCS 2208 (2001) $1338-1340$

12. Chinzei, K., Hata, N., Jolesz, F. A., Kikinis, R.: MR compatible surgical assist robot: System integration and preliminary feasibility study. Medical Image Computing and Computer-Assisted Intervention - MICCAI 2000, LNCS 1935 (2000)921-930

13. Jolesz, F.A., Hynynen, K.: Magnetic resonance image-guided focused ultrasound surgery. Cancer J., 8 (2002) S100-S112

14. Welch, E. B., Manduca, A., Grimm, R. C., Ward, H. A., Jack, Jr. C. R. : Spherical navigator echoes for full 3D rigid body motion measurement in MRI. Magnet. Reson. Med. 47 (2002) 32-41 